\title{
Some aspects of the anemia of chronic disorders modeled and analyzed by petri net based approach
}

\author{
Dorota Formanowicz • Andrea Sackmann • \\ Adam Kozak · Jacek Błażewicz • Piotr Formanowicz
}

Received: 17 August 2010/Accepted: 20 December 2010/Published online: 8 January 2011

(C) The Author(s) 2011. This article is published with open access at Springerlink.com

\begin{abstract}
Anemia of chronic disorders is a very important phenomenon and iron is a crucial factor of this complex process. To better understand this process and its influence on some other factors we have built a mathematical model of the human body iron homeostasis, which possibly most exactly would reflect the metabolism of iron in the case of anemia and inflammation. The model has been formulated in the language of Petri net theory, which allows for its simulation and precise analysis. The obtained results of the analysis of the model's behavior, concerning the influence of anemia and inflammation on the transferrin receptors, and hepcidin concentration changes are the valuable complements to the knowledge following from clinical research. This analysis is one of the first attempts to investigate properties and behavior of a not fully understood biological system on a basis of its Petri net based model.
\end{abstract}

Keywords Iron · Hepcidin · Inflammation · Modeling · Petri net theory

\section{Introduction}

Iron balance is tenuous, and both iron deficiency and iron overload are deleterious. Iron homeostasis is orchestrated

D. Formanowicz $(\bowtie)$

Department of Clinical Biochemistry, Poznań University of Medical Sciences, Grunwaldzka 6, 60-780 Poznań, Poland e-mail: doforman@ump.edu.pl

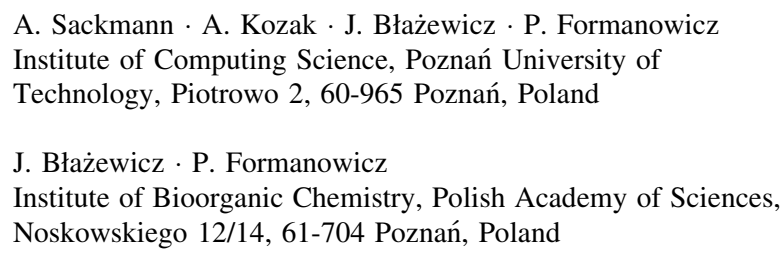

at the cellular as well as at the systemic level [1]. At the cellular level, iron homeostasis is aimed at controlling the iron uptake, storage and export as well as the intracellular iron management and distribution. At the systemic level, iron homeostasis aims to meet the erythroid demands and keep the iron stores replete. The recent discovery of hepcidin (the iron regulatory hormone) has provided a consistent model of the iron homeostasis that allows for a better understanding of the importance of sensors at the cellular level for iron needs, as well as of the pathophysiology of the hereditary iron metabolism disorders such as hemochromatosis and anemia of chronic disorders (ACD).

Iron deficiency is by far the most common hematological disorder encountered in general practice. The basic approach to its diagnosis and management is well established and outlined in most medical and hematological texts. However, the patients suffering from anemia very often are affected by other additional medical problems, such as bacterial and viral infections, an inflammation or malignant tumors. Thus, an assessment of the body iron status seems to be more complex issue. Such conditions mentioned above exhibit a blunted erythropoietin (EPO) response by erythroid precursors, a decreased red blood cell survival, abnormalities in both iron absorption and the retention of iron in the one-nuclear cells, and they restrict the availability of iron to the erythroid precursors in the bone marrow.

ACD is a common problem particularly in patients with end-stage renal disease and it is present in a vast majority of patients treated with maintenance hemodialysis. The immunological basis of an inflammatory process in dialysis was presented among others in [2-4].

Fortunately, the renal anemia has been shown to be effectively alleviated or reversed by recombinant human erythropoietin (rHuEPO) which increases the speed of 
erythropoiesis by many factors [5]. Doubling the erythropoietic activity would require a twofold iron demand. In such a state, the iron turnover will be a function of the iron stores and the erythropoietic activity of the bone marrow. The assessment of the iron requirements and an adequate iron supply are a prerequisite to an optimal response to rHuEPO.

A number of laboratory tests are employed to determine the iron status in patients receiving rHuEPO therapy, however, a valid method for the diagnosis of an iron deficiency anemia is still debatable. The iron availability may not keep pace with the iron demand for rHuEPOenhanced erythropoiesis. This situation is known as a functional iron deficiency.

Because of the complexity of this issue, our emphasis in this article is put on the better understanding of the phenomenon of ACD and its diagnosis. For this purpose, we built a model of the body iron homeostasis formulated in the language of Petri net theory, which is precise enough for a possible analysis and allows a simulation of the process. A Petri net is a well-known mathematical structure, which has found many applications in the analysis of technical systems of many types. The concept of Petri nets was proposed in the early 1960s by Carl A. Petri [6] in the area of technical systems as formalism suitable for modeling and the analysis of concurrent, asynchronous, distributed systems. With a rapid growth of computational biology in the last decade of the twentieth century, the structure of Petri nets was also used to model biological systems [7]. Since that time, Petri nets are being used for describing and analyzing various biological processes, e.g., metabolic pathways [8], signal transduction pathways [9], and gene-regulatory networks [10]. For an overview of the biological applications see, e.g., $[11,12]$. Generally, the advantages of using Petri nets for the required model are on the one hand their feature to represent the system behavior even when the biological process is not understood in every detail by combining different abstraction levels in one model [13]. And on the other hand, Petri nets comprise besides their firm mathematical foundation a visual representation of the model which enables a simulation of the system facilitating an intuitive comprehension of the biological pathways [14].

In the following, a very brief introduction to the Petri net theory is given. A more detailed overview of the theory can be found in $[15,16]$.

A Petri net is a directed graph composed of two kinds of nodes and arcs connecting some of them but only nodes of different types can be connected in this way. One kind of nodes is given by the places which model the passive system components like states or biological species and they are represented by circles. The second kind of nodes is called transitions standing for the active system components as events or biological reactions, which in graphical representation are depicted as rectangles. Thus, the net arcs describe the interaction between the active and the passive system elements. Besides these structural elements, a Petri net has an additional dynamic component given by tokens, which can flow through the net. Generally, the tokens reside in places indicating that the corresponding state is fulfilled or, in a biological context, the corresponding substance is present in the system. A possible token flow is defined by the net structure. According to the arc direction every place (transition) may have a set of preand post-transitions (pre- and post-places). If all pre-places of a transition are marked with tokens, this transition is enabled to fire, i.e., the corresponding event or reaction may take place if all its reactants or other necessary chemical substances are available. With its firing the transition removes tokens from all its pre-places and puts tokens to all its post-places, i.e., the reaction removes a token of each its precursors and produces a token of each its products, since the tokens indicate the presence of the substance. In general, the number of moved tokens depends on the corresponding arc weight. But with relation to the model presented in this paper, we consider only ordinary Petri nets, i.e., all arcs are weighted with one. Altogether, arcs connect an event with its pre-conditions, which must be fulfilled to trigger this event and with its post-conditions, which will be fulfilled by occurring of that event. A transition without pre-places, a so-called input transition, is not determined by the tokens of the net and thus the event modeled by an input transition may take place at every time. In the case that a condition must be fulfilled but the firing of an adjacent transition does not remove any tokens from the corresponding place, these nodes are connected via two converse arcs. In the model, they are represented by bi-directional arrows and are called read arcs. Generally, a place in a Petri net may carry any integer number of tokens, indicating different degrees of fulfillment or different quantities of the corresponding substance. Since the number of pre-places of a transition has not to be equal to the numbers of its post-places, the total number of tokens in the net is not conserved. The distribution of the tokens over all places is called the marking of the net and describes a certain system state. Correspondingly, the initial marking represents the system state before the firing of any transition.

\section{Materials and methods}

The model

To develop the Petri net model, at first all relevant biological interactions are translated into basic logical terms as 
implication, conjunction, disjunction, and negation. These logical terms are then unambiguously transformed into Petri net components. The graphical representation of a small Petri net example given in Fig. 1 serves as an illustration. A conjunction-coupled implication is given with $p 0 \wedge p 1 \Rightarrow p 2$, i.e., only if the places $p 0$ and $p 1$ are marked transition $t 2$ may fire. Its firing puts a token at place $p 2$. The two post-transitions of place $p 2, t 3$, and $t 4$, are in conflict, i.e., one token at place $p 2$ may enable both of these transitions but only one of them can fire. The firing of $t 3$ additionally depends on the marking of place $p 5$. If $p 5$ is not marked, a token on place $p 2$ leads to the firing of transition $t 4$ (as the only enabled post-transition), i.e., $p 2 \wedge \neg p 5 \Rightarrow p 4$. If there is a token on $p 5$, a non-deterministic behavior results since the corresponding subnet represents an exclusive disjunction, i.e., $p 2 \wedge p 5 \Rightarrow$ $p 3 \oplus p 4$.

Finally, an assembling of subnets built in that way gives the model. If there are some places without pre- or post-transitions in the net, some additional transitions are included representing the interface of the biological system to its surrounding (compare transitions $t 0, t 1, t 7$, and $t 8$ in Fig. 1). This modeling approach is introduced in [17].

According to this approach, a Petri net is built modeling the body iron homeostasis. This model contains 47 places ( $p$ ) and 57 transitions $(t)$, shown in Fig. 2. For the sake of clarity, nodes are only named with their IDs. The corresponding names of the places and transitions are listed in Tables 1 and 2 [18], respectively, and they are selfexplanatory formulated.

Our model has been precisely described recently in $[18,19]$ and with using the time intervals in [20]. In the presented model, we took into consideration the fact that anemia associated with a chronic inflammatory state is multi-factorial. During an inflammation, the body iron

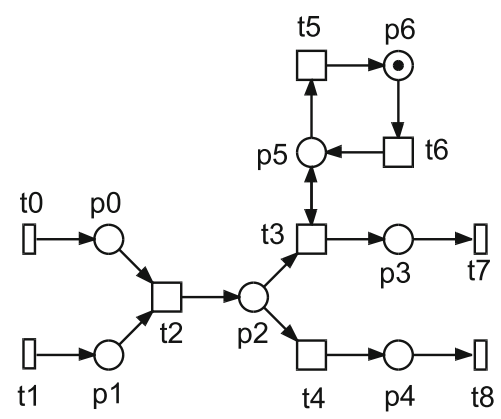

Fig. 1 A small Petri net containing seven places and nine transitions serving as an example. Input and output transitions are depicted as flat rectangles. The token of the initial marking is depicted by the dot on the place. For more details about it, its p-invariant and its three t-invariants, please see the text metabolism is changing. Iron is indispensable to living organisms; in vitro it is also conducive to bacterial growth [21]. Some bacteria, in fact, have an iron uptake system that allows for the direct uptake of iron by means of a surface TfR. Within this context, no free iron is available in the circulation to promote the replication of microorganisms [22]. In vivo, a number of mechanisms exist to limit the bacterial use of iron, notably an iron uptake via the reticuloendothelial system (the one-nuclear cells). So, an infectious and inflammatory disease commonly results a low serum iron concentration, which in consequence may lead to anemia.

Recently, it was found that hepcidin, a protein whose production is modulated in response to an inflammation, anemia, and hypoxia, is a link between anemia and inflammation [23]. Hepcidin, by binding to ferroportin (Fpn), appears to block the iron uptake in the small intestine and the release from the one-nuclear cells thereby decreasing the delivery of iron to the red blood cells precursors in the bone marrow [24].

In our model, a part of the network including correlations between the body iron and an inflammatory process was based on the Frazer and Anderson hypothesis [25]. According to this, the regulation of the hepcidin expression depends on the ratio of transferrin receptor 1 (TfR1) on the one-nuclear cells' surface to the HoloTf $\left(\mathrm{Fe}^{3+}\right)$ concentration in the serum. Hepatocyte surface HFE (the hemochromatosis protein) competes with HoloTf $\left(\mathrm{Fe}^{3+}\right)$ for the binding on surface TfR1, which is a TfR2 competitor. Unbound surface HFE and a higher concentration of the HoloTf $\left(\mathrm{Fe}^{3+}\right)$ TfR2 complex increase the hepatic hepcidin expression and its release. According to this model, an iron deficiency would lead to a decrease of the circulating HoloTf $\left(\mathrm{Fe}^{3+}\right)$, and the concentration of free surface TfR 1 s would increase, resulting in a decreasing fraction of the free surface HFE and in a lower concentration of the HoloTf $\left(\mathrm{Fe}^{3+}\right)$ TfR2 complex. This has been modeled in following way: HoloTf $\left(\mathrm{Fe}^{3+}\right)+\mathrm{TfR} 2$ ( $\left.t 47\right)$ leads via free HFE to a hepcidin increase ( $t 48)$ that leads to a decrease of Fpn (t36). A too small amount of Fpn avoids the iron transport out of the small intestine $(t 11)$ as well as the iron transport out of the one-nuclear cell $(t 28)$. If the iron level is low, there is more TfR1 than TfR2 (t46) and, therefore, there is no free HFE. In the model, the iron release out of the ferritin-connected store requires that there is no inflammation in the system ( $t 32$ in the one-nuclear cell and $t 44$ in the small intestine).

The analysis of the model

The developed model has to be verified by an analysis of the net. A general overview of analysis possibilities is 
Fig. 2 The Petri net modeling the body iron homeostasis. Nodes are labeled with their IDs; their corresponding names are listed in Table 1, and Table 2, respectively. Logical places are tinted in grey and avoid immoderate arc crossing. A logical node is defined by its ID and exists in multiple copies in the net, which are logically identical. Thus, this model is a connected Petri net. Functional or spatial subunits are marked by accordingly labeled ellipses

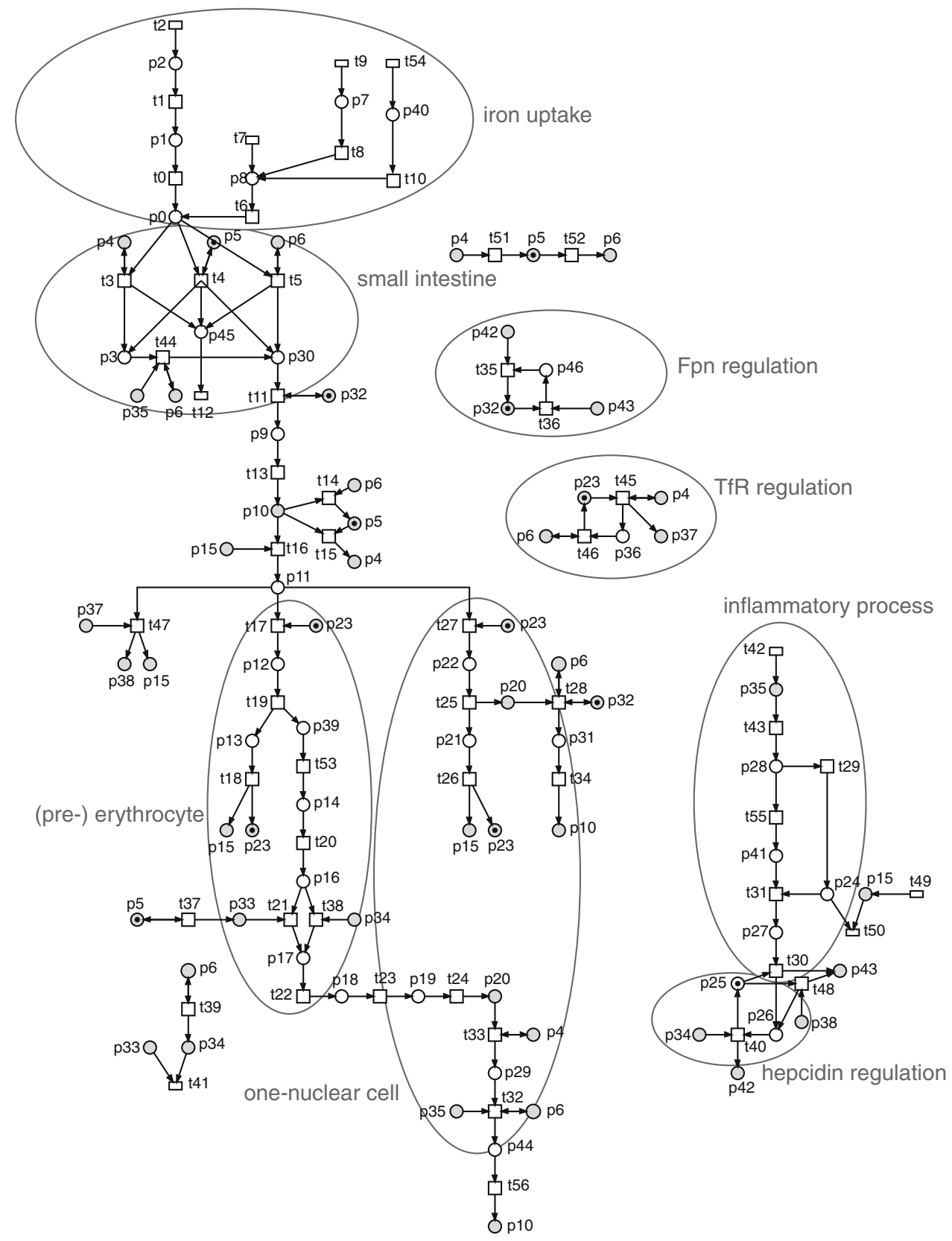

given, e.g., in $[13,25,26]$. Further explanations for the analysis of this particular Petri net model are given in $[18,27]$.

At first, some structural properties are calculated based on the net structure. A main part of the further model validation consists in the invariant analysis based on the incidence matrix [28]. Each entry of this (place $\times$ transition) matrix $C$ gives the token change on a particular place by firing of the respective transition. Thus, no information about node connections via read arcs is included in $C$. The transition matrix for the simple net shown in Fig. 1 is as follows (rows correspond to places and columns correspond to transitions):

$$
C=\left[\begin{array}{ccccccccc}
1 & 0 & -1 & 0 & 0 & 0 & 0 & 0 & 0 \\
0 & 1 & -1 & 0 & 0 & 0 & 0 & 0 & 0 \\
0 & 0 & 1 & -1 & -1 & 0 & 0 & 0 & 0 \\
0 & 0 & 0 & 1 & 0 & 0 & 0 & -1 & 0 \\
0 & 0 & 0 & 0 & 1 & 0 & 0 & 0 & -1 \\
0 & 0 & 0 & 0 & 0 & -1 & 1 & 0 & 0 \\
0 & 0 & 0 & 0 & 0 & 1 & -1 & 0 & 0
\end{array}\right]
$$

A p-invariant is calculated as a vector $y$ satisfying the equation $y \cdot C=0$ and it is called a minimal p-invariant if it does not contain any other p-invariant. Such an invariant provides a set of places over which the weighted sum of tokens is constant. As a clear example, consider the Petri 
Table 1 The names and IDs of the places of the model

\begin{tabular}{|c|c|c|c|}
\hline ID & Place name & ID & Place name \\
\hline 0 & LIP (in the small intestine) & 24 & IL-6 (cytokine) \\
\hline 1 & $\begin{array}{l}\mathrm{Hem}\left(\mathrm{Fe}^{2+}\right) \text { in the small } \\
\text { intestine }\end{array}$ & 25 & Less hepcidin \\
\hline 2 & Hem $\left(\mathrm{Fe}^{2+}\right)$ & 26 & Much hepcidin \\
\hline 3 & Ferritin $\left(\mathrm{Fe}^{3+}\right)$ & 27 & Much HAMP protein \\
\hline 4 & Fe serum high & 28 & Inflammatory process \\
\hline 5 & Fe serum medium & 29 & Ferritin $\left(\mathrm{Fe}^{3+}\right)$ \\
\hline 6 & Fe serum low & 30 & Available $\mathrm{Fe}^{2+}$ \\
\hline 7 & $\mathrm{Fe}^{3+}$ & 31 & $\begin{array}{l}\mathrm{Fe}^{2+} \text { in serum (the one- } \\
\text { nuclear cell) }\end{array}$ \\
\hline 8 & $\mathrm{Fe}^{2+}$ & 32 & Much Fpn \\
\hline 9 & $\mathrm{Fe}^{2+}$ in serum & 33 & Erythrocyte synthesis support \\
\hline 10 & $\mathrm{Fe}^{3+}$ in serum & 34 & Much EPO \\
\hline 11 & HoloTf $\left(\mathrm{Fe}^{3+}\right)$ & 35 & No inflammatory process \\
\hline 12 & HoloTf $\left(\mathrm{Fe}^{3+}\right)+\mathrm{TfR} 1$ & 36 & Less TfR1 \\
\hline 13 & ApoTf + TfR1 & 37 & TfR2 \\
\hline 14 & LIP in pre-erythrocyte & 38 & Free HFE \\
\hline 15 & ApoTf & 39 & $\mathrm{Fe}^{2+}$ \\
\hline 16 & $\operatorname{Hem}\left(\mathrm{Fe}^{2+}\right)$ & 40 & $\mathrm{Fe}^{3+}$ \\
\hline 17 & Erythrocyte & 41 & Much HFE protein \\
\hline 18 & $\begin{array}{l}\mathrm{Hem}\left(\mathrm{Fe}^{2+}\right) \text { in the one- } \\
\text { nuclear cell }\end{array}$ & 42 & Positive Fpn signal \\
\hline 19 & Free $\mathrm{Fe}^{2+}$ & 43 & Negative Fpn signal \\
\hline 20 & LIP & 44 & $\begin{array}{l}\mathrm{Fe}^{2+} \text { in serum (one-nuclear } \\
\text { cell) }\end{array}$ \\
\hline 21 & ApoTf + TfR1 & 45 & Mitochondrium/enzymes \\
\hline 22 & HoloTf $\left(\mathrm{Fe}^{3+}\right)+$ TfR1 & 46 & Less Fpn \\
\hline
\end{tabular}

Some of these places are equally named (e.g. $p 8$ and $p 39$ ), but they are identified as different nodes, because they were not defined as logical nodes [18]

LIP labile iron pool, HoloTf holotransferrin, ApoTf apotransferrin, $I L$ 6 interleukin-6, HAMP hepcidin antimicrobial peptide, Fpn ferroportin, TfR, TfR1, TfR2 transferrin receptors, EPO erythropoietin, $H F E$ the hemochromatosis protein

net in Fig. 1. It has one minimal p-invariant given by the vector $(0,0,0,0,0,1,1)$, i.e., the places $p 5$ and $p 6$ form such an invariant and, therefore, the token placed on $p 6$ in the initial marking circulates only between those two places (by firing of transitions $t 5$ and $t 6$ ). A required number of tokens (here one) has to be put in the initial marking at a place of each p-invariant to ensure that this subnet may contribute to the system behavior.

The iron homeostasis model contains four minimal p-invariants. Hence, the places $F e$ serum medium ( $p 5)$, Much Fpn (p32), Less hepcidin (p25), and TfR1 (p23) are marked with tokens already in the initial marking. Note that the tokens are put at one place of each p-invariant in a way representing an inactive state of the model. That means a medium level of iron, a high level of Fpn, a low level of hepcidin, and a higher amount of TfR 1 compared with the amount of TfR2 is assumed to be the physiologically normal state of the system. These tokens contained in the initial marking of the net will circulate only between places of each minimal p-invariant. For example, the first p-invariant consists of the places $\mathrm{Fe}$ serum high (p4), $\mathrm{Fe}$ serum medium ( $p 5)$, and Fe serum low ( $p 6)$. Thus, the mentioned token will only circulate between those three places, indicating the level of iron in the serum. The second and the third minimal p-invariants, both consist of two places, which are less hepcidin (p25) and much hepcidin (p26), and much Fpn (p32), and less Fpn (p46), respectively. Each of the two tokens circulating between those places indicates the changed available quantity of hepcidin and Fpn, respectively. The fourth minimal p-invariant indicates by its token the form in which TfR 1 is present in the system. It contains the six places HoloTf $\left(\mathrm{Fe}^{3+}\right)+$ TfR1 (p12), ApoTf + TfRl (p13), ApoTf + TfR1 (p21), HoloTf $\left(\mathrm{Fe}^{3+}\right)+$ TfRl (p22), TfRl (p23), and less TfRl (p36). To distinguish these places see Fig. 2.

The minimal t-invariants of a net are calculated as vectors $x$ satisfying the equation $C \cdot x=0$ and not containing any other such a vector and give a multiset of transitions which have altogether a zero effect on the marking, i.e., if all of them have fired the required number of times, a given marking is reproduced. Since the initial marking stands for that system state assumed as the normal state of the body, the t-invariants are a set of processes which lead again to that normal state after iron has been absorbed or other reactions have taken place.

The example net shown in Fig. 1 again should serve as an illustration. It has three minimal t-invariants: given by vectors $(0,0,0,0,0,1,1,0,0),(1,1,1,1,0,0,0,1,0)$, and $(1,1,1,0,1,0,0,0,1)$. It means that the first of them contains the transitions $t 5$ and $t 6$ (representing the abovementioned circulation of the token in the p-invariant). The second one consists of $t 0, t 1, t 2, t 4$, and $t 8$ and the third minimal t-invariant includes $t 0, t 1, t 2, t 3$, and $t 7$. The latter one is not feasible since it takes not into account the read arc between $t 3$ and the empty place $p 5$. A feasible $\mathrm{t}$-invariant is a minimal $\mathrm{t}$-invariant such that all transitions not belonging to this invariant. The reason that a minimal t-invariant is not a feasible t-invariant is an occurrence of read arcs, which are not reflected in the net incidence matrix (cf. [17, 18]). Thus, a processing of the minimal t-invariants combines these ones containing such connections with invariants providing tokens at these places see [17]. This combination leading to feasible t-invariants results for the example net in the t-invariants: firstly, $t 5$ and $t 6$, secondly, $t 0, t 1, t 2, t 4$, and $t 8$, and thirdly, $t 0, t 1, t 2, t 3$, $t 5, t 6$, and $t 7$.

Since every possible invariant (i.e., p- and t-invariant) can be built as non-negative linear combination of minimal 
Table 2 The names and IDs of the transitions of the model [18]

$H O$ heme oxygenase, LIP labile iron pool, IRP iron responsive protein, DMT1/NRAMP2/DCT1 divalent metal transporter-1 (natural resistance-associated macrophage protein- 2 or divalent cation transporter-1), Dcytb duodenal cytochrome b, Heph hephaestin, ApoTf apotransferrin, $R M E$ receptormediated endocytosis, $T f R$, $T f R 1, T f R 2$ transferrin receptors, Nramp1 natural resistanceassociated macrophage protein1, IL-6 interleukin-6, HAMP hepcidin antimicrobial peptide, $C p$ ceruloplasmin, $F p n$ ferroportin, $E P O$ erythropoietin, HoloTf holotransferrin, HFE the hemochromatosis protein

\begin{tabular}{|c|c|c|c|}
\hline ID & Transition name & ID & Transition name \\
\hline 0 & Oxygenation via $\mathrm{HO}$ & 29 & IL-6 increase \\
\hline 1 & Transport into the small intestine (transporter $\mathrm{X}$ ) & 30 & Hepcidin increase (expressed in the liver) \\
\hline 2 & Hem $\left(\mathrm{Fe}^{2+}\right)$ uptake & 31 & HAMP expression \\
\hline 3 & LIP split (IRP inactive) & 32 & $\left(\mathrm{Fe}^{2+}\right)$ release \\
\hline 4 & LIP split & 33 & Store (apoferritin) (IRP) \\
\hline 5 & LIP split (IRP active) & 34 & Oxidation (Cp) \\
\hline 6 & $\begin{array}{l}\text { Transport into the small intestine } \\
\text { (DMT1/NRAMP2/DCT1) }\end{array}$ & 35 & Fpn increase \\
\hline 7 & $\left(\mathrm{Fe}^{2+}\right)$ uptake & 36 & Fpn decrease/inhibition \\
\hline 8 & Reduction by HCL & 37 & Positive signal \\
\hline 9 & $\left(\mathrm{Fe}^{3+}\right)$ uptake & 38 & Erythrocyte synthesis \\
\hline 10 & Reduction by Dcytb & 39 & Signal for EPO synthesis in the kidneys \\
\hline 11 & Transport out of the small intestine & 40 & Hepcidin inhibition \\
\hline 12 & Loss & 41 & EPO decrease \\
\hline 13 & Oxidation (Heph) & 42 & Normal state \\
\hline 14 & Increase of low serum Fe level & 43 & Inflammatory process \\
\hline 15 & Increase of medium serum $\mathrm{Fe}$ level & 44 & $\mathrm{Fe}^{2+}$ release \\
\hline 16 & Binding of ApoTf $+\mathrm{Fe}^{3+}$ & 45 & TfR1 inhibition (RNA degradation via IRP) \\
\hline 17 & Endocytosis in pre-erythrocyte (RME) (TfR1) & 46 & TfR1 synthesis (RNA stabilization via IRP) \\
\hline 18 & Exocytosis + cleavage & 47 & HoloTf $\left(\mathrm{Fe}^{3+}\right)+\mathrm{TfR} 2$ \\
\hline 19 & Cleavage by low $\mathrm{pH}$ & 48 & Hepcidin increase (expressed in the liver) \\
\hline 20 & $\begin{array}{l}\text { Hem synthesis (ferrochelatase and } \\
\text { protoporphyrin IX) }\end{array}$ & 49 & Apo Tf synthesis \\
\hline 21 & Erythrocyte synthesis & 50 & Apo Tf synthesis inhibition \\
\hline 22 & Phagocytosis in the one-nuclear cell & 51 & Fe consumption \\
\hline 23 & Oxygenation & 52 & Fe consumption \\
\hline 24 & Transport via Nramp1 & 53 & $\begin{array}{l}\text { Transport into cytosol } \\
\text { (DMT1/NRAMP2/CDT1) }\end{array}$ \\
\hline 25 & Cleavage by low $\mathrm{pH}$ & 54 & $\left(\mathrm{Fe}^{3+}\right)$ uptake parenteral \\
\hline 26 & Exocytosis + cleavage & 55 & HFE expression \\
\hline 27 & Endocytosis in the one-nuclear cell (RME)(TfR1) & 56 & Oxidation (Cp) \\
\hline 28 & Transport out of the one-nuclear cell & & \\
\hline
\end{tabular}

invariants in most cases it is sufficient to consider only the minimal ones. So, in our analysis only the minimal invariants are taken into account. Moreover, we focused on minimal t-invariants, since the analysis of them gives the most interesting biological results (at least in the case of our system).

In the model of the human body iron homeostasis, a processing of the 45 minimal t-invariants of the net leads to 85 feasible t-invariants. A specification of the feasible t-invariants can be found in Table 4 . The model is covered by feasible t-invariants, i.e., each transition is contained in such a t-invariant. Therefore, each modeled event takes place in the basic behavior of the net since the feasible t-invariants characterize this behavior [17, 28]. For the purpose of model verification, it has been checked and at last confirmed that each feasible t-invariant has a biological equivalent, see [18].
Based on the feasible t-invariants it is decidable for each pair of transitions if they occur only together with each other in all of the feasible t-invariants or, if that is not the case, if they occur only in different feasible t-invariants. Following this information, the so-called MCT sets are built which consist of the processes linked with each other. In other words, the MCT-sets are calculated in such a way that two transitions belong to the same set if and only if they are elements of exactly the same feasible t-invariants. Transition which is not contained in any t-invariant form a single-element MCT-set. These sets support the verification of a biological meaning of feasible t-invariants (cf. $[17,18])$. The MCT sets can be determined using the following simple algorithm:

Step 1. Compute $I\left(t_{i}\right)$, i.e., sets of t-invariants that share transition $t_{i}$, for all t-invariants $t_{i}$, e.g., create 
Table 3 The MCT-sets of the model and their biological meaning
LIP labile iron pool, IL-6 interleukin-6, EPO erythropoietin, Fpn ferroportin, $T f R$ transferrin receptor, $H F E$ hemochromatosis protein

\begin{tabular}{lll}
\hline MCT-set & Contained transitions & Biological meaning \\
\hline 1 & $0,1,2$ & Hem $\left(\mathrm{Fe}^{2+}\right)$ uptake \\
2 & $4,11,12,13,14,44,52$ & Iron transport through the small intestine \\
3 & $5,15,51$ & Iron level changes from low to high and decreases again \\
4 & 8,9 & $\left(\mathrm{Fe}^{3+}\right)$ uptake \\
5 & 10,54 & Parenteral $\left(\mathrm{Fe}^{3+}\right)$ uptake \\
6 & $17,18,19,20,22,23,24,53$ & Iron pathway through the erythrocyte \\
7 & $25,26,27$ & Iron pathway through the one-nuclear cell \\
8 & 28,34 & Iron from the LIP to the serum \\
9 & 29,43 & Inflammation and IL-6 increase \\
10 & $30,31,55$ & Inflammation caused increase of hepcidin \\
11 & $32,33,56$ & Iron from LIP to store and its release \\
12 & $35,36,40$ & Hepcidin decrease caused by much EPO, Fpn increase \\
13 & $45,46,47,48$ & TfR2 and free HFE caused hepcidin increase \\
14 & 49,50 & ApoTf decrease caused by IL-6 increase \\
\hline
\end{tabular}

empty set for each transition and add t-invariants by iteration over all transitions feasible t-invariants.

Step 2. Group equal sets $I\left(t_{i}\right)$, e.g., sort these sets lexicographically.

Step 3. Create MCT-sets corresponding to grouped sets obtained in Step 2, if a set is empty, then corresponding transition creates separate MCT-set.

Example: if the result of Step 1 is: $I\left(t_{1}\right)=\left\{y_{2}, y_{3}, y_{7}\right\}$, $I\left(t_{2}\right)=\left\{y_{1}, y_{3}, y_{7}\right\}, I\left(t_{3}\right)=\left\{y_{2}, y_{3}, y_{7}\right\}, I\left(t_{4}\right)=\left\{y_{1}, y_{3}, y_{7}\right\}$, $I\left(t_{5}\right)=\left\{y_{2}, y_{3}, y_{7}\right\}, I\left(t_{6}\right)=\varnothing, I\left(t_{7}\right)=\varnothing, \ldots, I\left(t_{|T|}\right)=\varnothing$, where $y_{i}$ is a feasible t-invariant and $|\mathrm{T}|$ is the number of transitions, then the result of Step 2 is: $G=\left\{\left\{I\left(t_{1}\right), I\left(t_{3}\right)\right.\right.$, $\left.\left.I\left(t_{5}\right)\right\},\left\{I\left(t_{2}\right), I\left(t_{4}\right)\right\}, I\left(t_{6}\right), I\left(t_{7}\right), \ldots, I\left(t_{|T|}\right)\right\}$ and the result of Step 3 is: $\mathrm{MCT}_{1}=\left\{t_{1}, t_{3}, t_{5}\right\}, \mathrm{MCT}_{2}=\left\{t_{2}, t_{4}\right\}, \mathrm{MCT}_{3}=$ $\left\{t_{6}\right\}, \mathrm{MCT}_{4}=\left\{t_{7}\right\}, \ldots, \mathrm{MCT}_{|G|}=\left\{t_{|T|}\right\}$.

The MCT-sets of the example net in Fig. 1 should illustrate this grouping of transitions. Here, the net contains four MCT-set. They are given after an examination of the three feasible t-invariants named in the text above. Thus, the sets are: MCT-set $1=\{t 5, t 6\}$; MCT-set $2=\{t 0, t 1$, $t 2\}$; MCT-set $3=\{t 3, t 7\}$; MCT-set $4=\{t 4, t 8\}$. The invariants can, therefore, be expressed in terms of MCTsets. Generally, the transitions remaining as single once after the grouping mentioned above, represent the so-called trivial MCT-sets. In the following, they will be named as single transitions.

The MCT-sets of the homeostasis model are given in Table 3. They are used also for Table 4 listing the feasible t-invariants (in this table the transitions composing each invariant and MCT-sets whose element a given invariant is also listed).

Based on the information given in Table 4, it is deducible which of the biological processes are independent of each other. In order to epitomize the information about the feasible t-invariants in a more compact way, the feasible t-invariants are grouped based on their similarities in the so-called t-clusters [29]. Figure 3 [18] gives the dendrogram of the ten t-clusters of the 85 feasible t-invariants built using various clustering approaches. The dendrograms shown in parts (a)-(d) of the figure are results of application of standard clustering approaches, i.e., UPGMA-based approach and with an accordance of $65 \%$, complete linkage with Euclidean distance metric, single linkage with percent disagreement metric and weighted pair group average with Euclidean distance metric, respectively. Clusters obtained using UPGMA method have been a base for grouping clusters obtained with the three remaining approaches, i.e., accordance in these approaches have been adjusted for clusters shown in part (a) for a comparison of the results. In addition, in part (e) of Fig. 3 there is shown a dendrogram obtained using some non-standard clustering approach, which we call pathwaybased approach, where feasible t-invariants having the same starting and ending transitions are considered as elements of the same cluster. As it can be noticed, the dendrograms shown in parts (a)-(d) of the figure are very similar to each other. The clusters obtained in all these cases are the same and the hierarchies differ not considerable.

The most important conclusion following from these results is that various classical clustering methods give the same t-clusters which can be used for an analysis of the biological properties of the net.

Part (e) of Fig. 3 shows the results of a clustering which are considerably different from those shown in parts (a)-(d). First of all, the number and the composition of the clusters differ from the previous one. The clusters hierarchy is also different. The reason is that the applied clustering procedure is based on a completely different idea. 
Table 4 The feasible t-invariants of the model

\begin{tabular}{|c|c|c|c|}
\hline t-cluster & $\begin{array}{l}\text { Feasible } \\
\text { t-invariant }\end{array}$ & MCT-sets & Transitions \\
\hline \multirow[t]{16}{*}{1} & 61 & $2,3,5,6,11$ & $3,6,16,38,39,42$ \\
\hline & 60 & $2,3,5,6,11$ & $6,16,38,39,42$ \\
\hline & 59 & $2,3,4,6,11$ & $3,6,16,38,39,42$ \\
\hline & 58 & $2,3,4,6,11$ & $6,16,38,39,42$ \\
\hline & 57 & $2,3,6,11$ & $3,6,7,16,38,39,42$ \\
\hline & 56 & $2,3,6,11$ & $6,7,16,38,39,42$ \\
\hline & 49 & $2,3,5,6,11$ & $3,6,16,21,37,42$ \\
\hline & 48 & $2,3,5,6,11$ & $6,16,21,37,42$ \\
\hline & 47 & $2,3,4,6,11$ & $3,6,16,21,37,42$ \\
\hline & 46 & $2,3,4,6,11$ & $6,16,21,37,42$ \\
\hline & 45 & $2,3,6,11$ & $3,6,7,16,21,37,42$ \\
\hline & 44 & $2,3,6,11$ & $6,7,16,21,37,42$ \\
\hline & 55 & $1,2,3,6,11$ & $3,16,38,39,42$ \\
\hline & 54 & $1,2,3,6,11$ & $16,38,39,42$ \\
\hline & 43 & $1,2,3,6,11$ & $3,16,21,37,42$ \\
\hline & 42 & $1,2,3,6,11$ & $16,21,37,42$ \\
\hline \multirow[t]{16}{*}{2} & 65 & $2,3,5,6,8$ & $3,6,16,38,39,42$ \\
\hline & 64 & $2,3,4,6,8$ & $3,6,16,38,39,42$ \\
\hline & 63 & $2,3,6,8$ & $3,6,7,16,38,39,42$ \\
\hline & 53 & $2,3,5,6,8$ & $3,6,16,21,37,42$ \\
\hline & 52 & $2,3,4,6,8$ & $3,6,16,21,37,42$ \\
\hline & 51 & $2,3,6,8$ & $3,6,7,16,21,37,42$ \\
\hline & 21 & $2,5,6,8$ & $6,16,38,39,42$ \\
\hline & 20 & $2,4,6,8$ & $6,16,38,39,42$ \\
\hline & 19 & $2,6,8$ & $6,7,16,38,39,42$ \\
\hline & 17 & $2,5,6,8$ & $6,16,21,37,42$ \\
\hline & 16 & $2,4,6,8$ & $6,16,21,37,42$ \\
\hline & 15 & $2,6,8$ & $6,7,16,21,37,42$ \\
\hline & 62 & $1,2,3,6,8$ & $3,16,38,39,42$ \\
\hline & 50 & $1,2,3,6,8$ & $3,16,21,37,42$ \\
\hline & 18 & $1,2,6,8$ & $16,38,39,42$ \\
\hline & 14 & $1,2,6,8$ & $16,21,37,42$ \\
\hline \multirow[t]{8}{*}{3} & 85 & $2,3,5,12,13$ & $6,16,39,42$ \\
\hline & 84 & $2,3,5,12,13$ & $3,6,16,39,42$ \\
\hline & 83 & $2,3,4,12,13$ & $6,16,39,42$ \\
\hline & 82 & $2,3,4,12,13$ & $3,6,16,39,42$ \\
\hline & 81 & $2,3,12,13$ & $6,7,16,39,42$ \\
\hline & 80 & $2,3,12,13$ & $3,6,7,16,39,42$ \\
\hline & 79 & $1,2,3,12,13$ & $16,39,42$ \\
\hline & 78 & $1,2,3,12,13$ & $3,16,39,42$ \\
\hline \multirow[t]{8}{*}{4} & 41 & $2,3,5,9,10,12$ & $3,6,39,42$ \\
\hline & 40 & $2,3,4,9,10,12$ & $3,6,39,42$ \\
\hline & 39 & $2,3,9,10,12$ & $3,6,7,39,42$ \\
\hline & 13 & $2,5,9,10,12$ & $6,39,42$ \\
\hline & 12 & $2,4,9,10,12$ & $6,39,42$ \\
\hline & 11 & $2,9,10,12$ & $6,7,39,42$ \\
\hline & 38 & $1,2,3,9,10,12$ & $3,39,42$ \\
\hline & 10 & $1,2,9,10,12$ & 39,42 \\
\hline
\end{tabular}

Table 4 continued

\begin{tabular}{|c|c|c|c|}
\hline t-cluster & $\begin{array}{l}\text { Feasible } \\
\text { t-invariant }\end{array}$ & MCT-sets & Transitions \\
\hline \multirow[t]{8}{*}{5} & 73 & $2,3,5,7,11$ & $3,6,16,42$ \\
\hline & 72 & $2,3,5,7,11$ & $6,16,42$ \\
\hline & 71 & $2,3,4,7,11$ & $3,6,16,42$ \\
\hline & 10 & $2,3,4,7,11$ & $6,16,42$ \\
\hline & 69 & $2,3,7,11$ & $3,6,7,16,42$ \\
\hline & 68 & $2,3,7,11$ & $6,7,16,42$ \\
\hline & 67 & $1,2,3,7,11$ & $3,16,42$ \\
\hline & 66 & $1,2,3,7,11$ & 16,42 \\
\hline \multirow[t]{8}{*}{6} & 77 & $2,3,5,7,8$ & $3,6,16,42$ \\
\hline & 76 & $2,3,4,7,8$ & $3,6,16,42$ \\
\hline & 75 & $2,3,7,8$ & $3,6,7,16,42$ \\
\hline & 25 & $2,5,7,8$ & $6,16,42$ \\
\hline & 24 & $2,4,7,8$ & $6,16,42$ \\
\hline & 23 & $2,7,8$ & $6,7,16,42$ \\
\hline & 74 & $1,2,3,7,8$ & $3,16,42$ \\
\hline & 22 & $1,2,7,8$ & 16,42 \\
\hline \multirow[t]{9}{*}{7} & 37 & $2,3,5$ & $3,6,37,39,41,42$ \\
\hline & 36 & $2,3,4$ & $3,6,37,39,41,42$ \\
\hline & 35 & 2,3 & $3,6,7,37,39,41,42$ \\
\hline & 33 & $2,3,5$ & $3,6,42$ \\
\hline & 29 & $2,3,5$ & 6,42 \\
\hline & 32 & $2,3,4$ & $3,6,42$ \\
\hline & 28 & $2,3,4$ & 6,42 \\
\hline & 31 & 2,3 & $3,6,7,42$ \\
\hline & 27 & 2,3 & $6,7,42$ \\
\hline \multirow[t]{6}{*}{8} & 9 & 2,5 & $6,37,39,41,42$ \\
\hline & 5 & 2,5 & 6,42 \\
\hline & 8 & 2,4 & $6,37,39,41,42$ \\
\hline & 7 & 2 & $6,7,37,39,41,42$ \\
\hline & 4 & 2,4 & 6,42 \\
\hline & 3 & 2 & $6,7,42$ \\
\hline \multirow[t]{5}{*}{9} & 34 & $1,2,3$ & $3,37,39,41,42$ \\
\hline & 30 & $1,2,3$ & 3,42 \\
\hline & 26 & $1,2,3$ & 42 \\
\hline & 6 & 1,2 & $37,39,41,42$ \\
\hline & 2 & 1,2 & 42 \\
\hline 10 & 1 & 9,14 & 42 \\
\hline
\end{tabular}

Table 5 shows the feasible t-invariants composing every of the t-clusters obtained with this method and also corresponding input and output transitions. They are the starting and ending transitions of a feasible t-invariant, on which the grouping procedure is based. Table 6 contains a comparison of the results of pathway-based clustering and the classical approaches. There are shown the numbers of common feasible t-invariants for all pairs of classical and non-classical t-clusters. 

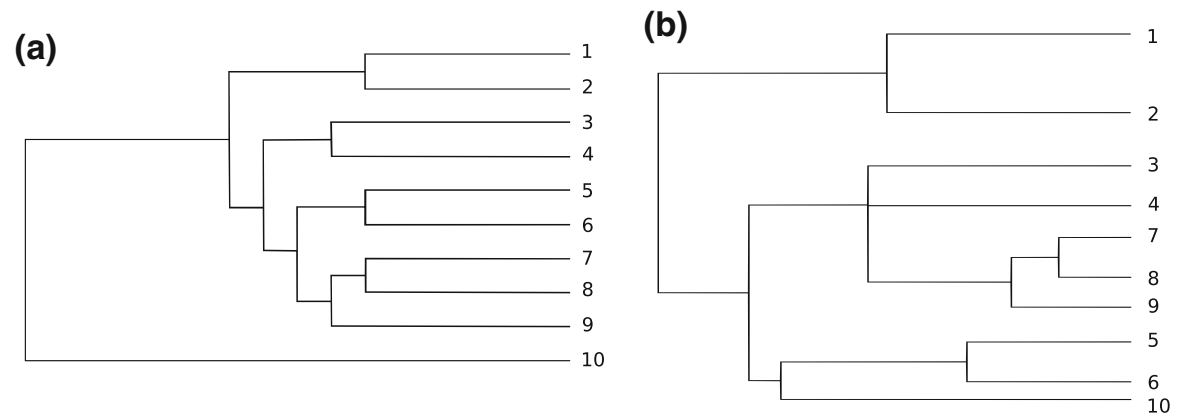

(c)

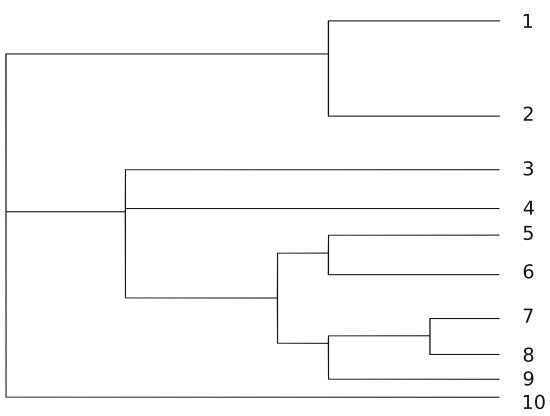

(d)

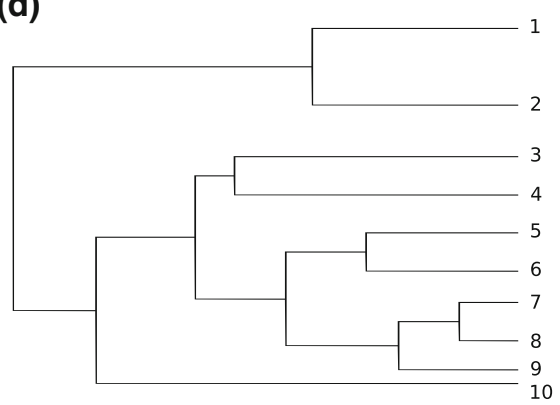

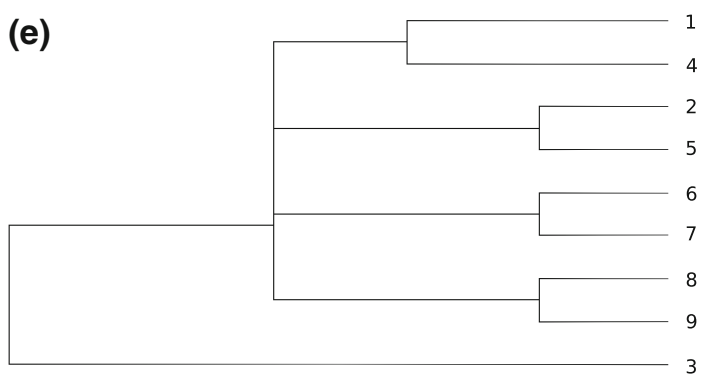

Fig. 3 Dendrograms and comparison of clustering methods. Numbers on the right indicate the number of cluster. In parts $\mathbf{a}-\mathbf{d}$, there are the same t-clusters 1-10 listed in Table 4-these clusters were computed using classical clustering methods based on size of common transition subsets. Clusters obtained in part a became a base for grouping clusters in b-d (accordance in $\mathbf{b}-\mathbf{d}$ was adjusted for clusters obtained in a for better comparison of hierarchy); a UPGMA-based clustering with an accordance of $65 \%, \mathrm{~b}$ complete linkage with Euclidean distances metric, $\mathbf{c}$ single linkage with percent disagreement metric, d weighted pair group average with Euclidean distances metric. All clustered t-invariants were represented as 57-dimensional vectors (transitions 0-56) where each position is equal to 0 or 1 depending on existence of a transition in given feasible t-invariant. In part $\mathbf{e}$, there are 9 clusters obtained from pathway-based method with transitions listed in Table 5. Each cluster was represented as 9-dimensional vector (6 different input transitions and 3 different output transitions) where each position is equal to 0 or 1 depending on existence of input/ output transition in given cluster

Table 5 List of t-invariants in clusters obtained from pathway-based clustering (all feasible t-invariants in a cluster have the same sets of input and output transitions)

\begin{tabular}{llll}
\hline T-cluster & Feasible t-invariant & Input transitions & Output transitions \\
\hline 1 & $81,80,75,69,68,63,57,56,51,45,44,39,31,27,23,19,15,11,3$ & 7,42 & 12 \\
2 & 7,35 & 42 & 41,12 \\
3 & 1 & 42,49 & 50 \\
4 & $85,84,77,73,72,65,61,60,53,49,48,41,33,29,25,21,17,13,5$ & 54,42 & 12 \\
5 & 9,37 & 54,42 & 41,12 \\
6 & $83,82,76,71,64,59,58,52,47,46,40,32,28,24,20,16,12,70,4$ & 9,42 & 12 \\
7 & 8,36 & 9,42 & 41,12 \\
8 & $79,78,74,67,66,62,55,54,50,43,42,38,30,26,22,18,14,10,2$ & 2,42 & 12 \\
9 & 6,34 & 2,42 & 41,12 \\
\hline
\end{tabular}


It seems that the non-classical t-clusters (i.e., the ones obtained using pathway-based clustering) are difficult to interpret from biological point of view, so in the further analysis the classical ones will be used.

\section{Results and discussion}

The main goal of our research was the formulation of the model, described above, which possibly most exactly would reflect the metabolism of the iron in the case of anemia and inflammation. In addition, on the basis of the analysis of the model's behavior we have attempted to make some observations concerning transferrin receptor and hepcidin.

\section{Transferrin receptor}

To the best of our knowledge, there is still no single biochemical parameter that is sensitive or specific enough to completely describe the distribution of iron in the human body. The routinely used laboratory tests, such as serum iron, total iron-binding capacity, transferrin saturation and serum ferritin are good indicators of the iron available for the erythropoiesis and for the iron stores but they are considerably influenced by a number of non-related conditions, e.g., the acute phase reactions, which may complicate the clinical interpretation of their results and create an inaccurate picture of the body iron status [30]. In this situation, one of the proposed parameter that could be useful enough for the body iron assessment is the soluble transferrin receptor (sTfR) whose evaluation is an indirect measure of the quantity of the membrane transferrin receptor (TfR) [31]. This assumption is based principally on the observed correlation between the concentration of the serum receptor molecule complexes with the transferrin and the ferrokinetic measurements of the erythron transferrin uptake [32, 33].

On the basis of the analysis of the models' behavior, we have attempted to check the influence of an inflammatory process on the level of TfR, i.e., TfR1 as well as TfR2. We have verified that an inflammatory process does not influence the TfR1 and TfR2 levels (no t-invariants contain both, the inflammatory process $(t 43)$ in MCT-set 9 in t-clusters 4 and 10 and the TfR regulation $(t 45, t 46)$ in MCT-set 13 in t-cluster 3). That means that there is no connection between these two processes in the basic behavior of the model.

We have also tried to check whether EPO bears on the TfR concentration. In our model the TfR regulation was dependent partially on an increased EPO level. We have disclosed that in every t-invariant, which includes the TfR regulation $(t 45, t 46)$, i.e., those of t-cluster 3 , there occurs
EPO production ( $t 39$ ), but on the other hand, EPO production also appears in t-invariants in which TfR is not regulated, namely in t-cluster 4 and partly in t-clusters 1 and 2 . So, in the model the TfR regulation is correlated to an increased EPO level. On this basis, we can suggest that EPO, and also a supplementation of rHuEPO which contributes to an increase of the serum EPO, may influence the TfR concentration and because of this the usefulness of sTfR for assessment of the body iron status in patients supplemented with rHuEPO has a questionable value. Our results have supported some previous reports [34] that rHuEPO intravenous dose administration influences the sTfR serum concentration in hemodialyzed patients. Some of the researchers suggested [34] that the maintenance rHuEPO administration may not only enhance the erythropoiesis in the bone marrow but also may contribute to the serum sTfR concentration increase [35]. Moreover, some of them implied that EPO itself raises intracellular calcium [36] that may contribute to an acceleration of the TfR recycling. EPO may also regulate the rate of the truncation of TfR from the cell membrane [34]. Other authors infer that the circulating sTfR levels may be useful in monitoring the iron status of mHD patients if the doses of rHuEPO are maintained constantly in those patients who are likely to be in a steady state erythropoietic stimulation [37, 38].

Another purpose of our study is to explore the factors determining the TfR1 concentration. Basing on the behavior of our model, we ascertained that a main factor affecting the concentration of this receptor was the concentration of the iron in the serum. The serum iron concentration (high serum iron concentration causes a TfR1 decrease and a TfR2 increase ( $t 45)$ in MCT-set 13) mainly influences the TfR concentration (which is always independent of an inflammatory process represented in MCTset 9, as has been mentioned above). The low serum iron concentration results in a TfR 1 increasing ( $t 46)$. The level of the serum iron concentration mainly influences the TfR regulation. A changed level of the Tf receptors influences the level of free HFE and via that way (in the case of a high level of TfR2) an increase of the hepcidin synthesis and a decrease/inhibition of Fpn ( $t 45$ and $t 46$ are grouped in MCT-set 13 together with $t 47$ (free HFE) and $t 48$ (corresponding hepcidin increase with Fpn inhibition), see Table 3).

We have also attempted to verify the hypothesis that the TfR 1 concentration is a good marker of the erythropoietic activity in the human body. In our model if TfR1 is down regulated (included only in t-cluster 3 ), there is no iron uptake to pre-erythrocyte and, therefore, there is no hem synthesis ( $t 20)$ which is contained in MCT-set 6 and occurs only in the t-clusters 1 and 2 . Looking at this, we may suggest that the TfR 1 serum concentration is a good marker of the erythropoietic activity. 
Table 6 Comparison of common transitions between pathway-based clustering (horizontal clusters 1-9) and classical clustering methods (vertical clusters $1-10$ )

\begin{tabular}{|c|c|c|c|c|c|c|c|c|c|}
\hline & 1 & 2 & 3 & 4 & 5 & 6 & 7 & 8 & 9 \\
\hline 1 & 4 & & & 4 & & 4 & & 4 & \\
\hline 2 & 4 & & & 4 & & 4 & & 4 & \\
\hline 3 & 2 & & & 2 & & 2 & & 2 & \\
\hline 4 & 2 & & & 2 & & 2 & & 2 & \\
\hline 5 & 2 & & & 2 & & 2 & & 2 & \\
\hline 6 & 2 & & & 2 & & 2 & & 2 & \\
\hline 7 & 2 & 1 & & 2 & 1 & 2 & 1 & & \\
\hline 8 & 1 & 1 & & 1 & 1 & 1 & 1 & & \\
\hline 9 & & & & & & & & 3 & 2 \\
\hline 10 & & & 1 & & & & & & \\
\hline
\end{tabular}

Numbers in the table are sizes of common t-invariant subsets within clusters

\section{Hepcidin}

Another question, which we would like to answer, is: does the EPO concentration influence the hepcidin concentration? This issue is very important, particularly for the patients with an ESRD diagnosis with a diminished EPO production in the kidneys, an undergoing rHuEPO treatment. In our model, there is no connection between a lower EPO concentration and hepcidin. On the other hand, an increase of the EPO concentration ( $t 38)$ depends on a low serum iron concentration, thus a high EPO concentration (t39) results in a hepcidin decrease $(t 40)$ connected with an increase of the Fpn ( $t 35)$ level (the last two ones are transitions in MCT-set 12). So, on the basis of the behavior of our model it is difficult to say for certain that the serum EPO concentration influences the hepcidin concentration, but we suggest that it can be the case.

We have also tried to find how the concentration of hepcidin, HFE, and TfR is changing if there is a low serum Fe level (during an inflammatory process). Generally, the transition $t 52$ (iron consumption which leads to a low iron level) in MCT-set 2 participates in every t-invariant (except of the first one which does not represent an iron flow through the net). From the point of view of the t-invariant calculation, a low iron level is needed in each of the three ways through the small intestine characterized by the three iron levels: high, medium, and low. If the serum iron level is low there is much EPO production ( $t 38 / t 39)$ causing a hepcidin decrease ( $t 40$ in MCT-set 12) and a TfR1 increase. In this situation, TfR2 is not synthesized ( $t 46$, MCT-set 13) followed by no free HFE ( $t 47$, MCT-set 13), no corresponding hepcidin increase ( $t 48$, MCT-set 13$)$ and Fpn decrease ( $t 36$, MCT-set 12). In Table 7, the discussed biological conclusions following from the analysis of the model have been summarized.

We can conclude that the analysis of the behavior of our Petri net-based model of the body iron homeostasis allows us to have the new insight into this complex process. Such an analysis is an interesting and valuable complement to the clinical research. On the other hand, it can also suggest some properties of the analyzed process which has not been realized previously. Moreover, since the formal model of the analyzed phenomenon is built on the basis of the current knowledge in a particular area its analysis can possibly also lead to a revision of this knowledge in cases when the obtained results do not agree with those following from

Table 7 Summary of the biological conclusions following from the analysis of the model behavior

\begin{tabular}{|c|c|c|}
\hline No. & Observations & Biological conclusions \\
\hline 1 & $\begin{array}{l}\text { No t-invariants contain both, an inflammatory process, i.e., } t 43 \text { in } \\
\text { MCT-set } 9 \text { in t-clusters } 4 \text { and } 10 \text {, and the TfR regulation, i.e., } t 45 \\
\text { band } t 46 \text { in MCT-set } 13 \text { in t-cluster } 3\end{array}$ & $\begin{array}{l}\text { The inflammatory process does not influence the TfR } 1 \text { and TfR } \\
\text { levels. }\end{array}$ \\
\hline 2 & $\begin{array}{l}\text { In every t-invariant which includes the TfR regulation, i.e., } t 45 \text { and } \\
\text { t } 46 \text { in t-cluster } 3 \text { there occurs EPO production, i.e., } t 39 \text {. On the } \\
\text { other hand, } t 39 \text { also appears in t-invariants in which TfR is not } \\
\text { regulated, i.e., in t-cluster } 4 \text { and partly in t-clusters } 1 \text { and } 2 .\end{array}$ & $\begin{array}{l}\text { The TfR regulation was dependant partially on an increased EPO } \\
\text { level. }\end{array}$ \\
\hline 3 & $\begin{array}{l}\text { A level of Tf receptors influences the level of HFE and an increase of } \\
\text { the hepcidin synthesis and a decrease/inhibition of Fpn- } t 45 \text { and } \\
t 46 \text { are grouped in MCT-set } 13 \text { together with HFE, i.e., } t 47 \text { band } \\
\text { hepcidin increase, i.e., } t 48 .\end{array}$ & $\begin{array}{l}\text { A main factor affecting the concentration of TfR } 1 \text { is the } \\
\text { concentration of the iron in serum. }\end{array}$ \\
\hline 4 & $\begin{array}{l}\text { If TfR } 1 \text { is down regulated (included only in t-cluster } 3 \text { ), there is no } \\
\text { iron uptake to pre-erythrocyte and therefore there is no hem } \\
\text { synthesis, i.e., } t 20 \text {, which is contained in MCT-set } 6 \text { and occurs } \\
\text { only in the t-clusters } 1 \text { and } 2 .\end{array}$ & $\begin{array}{l}\text { The TfR } 1 \text { serum concentration is a good marker of the erythropoietic } \\
\text { activity. }\end{array}$ \\
\hline 5 & $\begin{array}{l}\text { Iron consumption leading to a low iron level, i.e., } t 52 \text { in MCT-set } 2 \text { is } \\
\text { included in every t-invariant except the first one, which does not } \\
\text { represent an iron flow through the net. }\end{array}$ & $\begin{array}{l}\text { A low iron level is needed in each of the ways through the small } \\
\text { intestine characterized by the three iron levels, i.e., high, medium, } \\
\text { and low. }\end{array}$ \\
\hline
\end{tabular}


Table 8 The associations between sTfR and selected inflammatory proteins obtained during laboratory tests from patients with anemia of chronic disorders

\begin{tabular}{llll}
\hline Variable & CRP & IL-1 & IL-6 \\
\hline sTfR & $r=-0.155$ & $r=0.0085$ & $r=-0.2005$ \\
& $p=0.297$ & $p=0.955$ & $p=0.177$ \\
\hline
\end{tabular}

$C R P$ C-reactive protein, $I L-1$ interleukin 1 , IL-6 interleukin 6 , $r$ Pearson's correlation coefficient, $p$ value of the significance level

classical experiments (but in this case the verification of the experiment results can be also necessary in same cases). For example, in [39] it was found that the serum concentration of TfR is probably not influenced by EPO concentration but from our model it follows the opposite conclusion. The relation between these two concentrations was not explicitly used for building the model, hence it follows from the knowledge which was implicitly the basis for the model construction. The disagreement between the experimental and the theoretical (i.e., the ones following from the analysis of the model) results suggests the necessity of a revision of the knowledge of the human body iron homeostasis process (since it leads to conclusions contradicting the clinical results) or a verification of the clinical experiments. In both cases, the analysis of the theoretical model of the process leads to clarifying some of its aspects and better understanding this complex biochemical phenomenon. Following this direction of research, recently we have performed the new more accurate laboratory tests (concerning only patients with anemia of chronic disorders) whose results show that the conclusions concerning the relationship between $\mathrm{TfR}$ and rHuEPO coming from our model are correct (see "Validation of the model").

\section{Validation of the model}

Materials and methods

In order to validate our model and verify whether the obtained results are confirmed in lab tests we used data obtained from 50 patients with end-stage renal disease, hemodialyzed in the Department of Nephrology, Transplantology and Internal Medicine, at Poznań University of Medical Sciences. To the analysis, we included only lab tests results from the patients suffering from anemia of chronic disorders and treated with rHuEPO. In every patient, the selected serum parameters, using ELISA-kits, like iron $(\mathrm{Fe})$, soluble transferrin receptor (sTfR) which concentration is proportional to the total concentration of cellular TfR, hepcidin, erythropoietin concentration (EPO) were assessed. For the evaluation of the inflammatory status, we examined the levels of selected acute phase proteins, i.e., C-reactive protein (CRP), Interleukin-1 (IL1), and Interleukin-6 (IL-6).

All tests were performed according to the manufacture's instruction. Ethical approval was obtained from the local ethics committee before the start of the study and all patients were asked for their verbal consent before the blood was taken.
Fig. 4 The scatter diagram (with histograms, i.e., the upper and the right panel, which are a graphical way of presenting the empirical distribution characteristics) shows the association between STfR and rHuEPO. It illustrates the strong positive correlation

( $r=0.37332, p=0.01)$, which suggests a strong relationship between these variables. The solid line indicates the linear regression, and the dotted linethe $95 \%$ confidence interval. $s T f R$ soluble transferrin receptor whose serum concentration is proportional to the total concentration of cellular TfR, $r H u E P O$ mean weekly dose of recombinant human erythropoietin, $r$ Pearson's correlation coefficient, $p$ value of the significance level
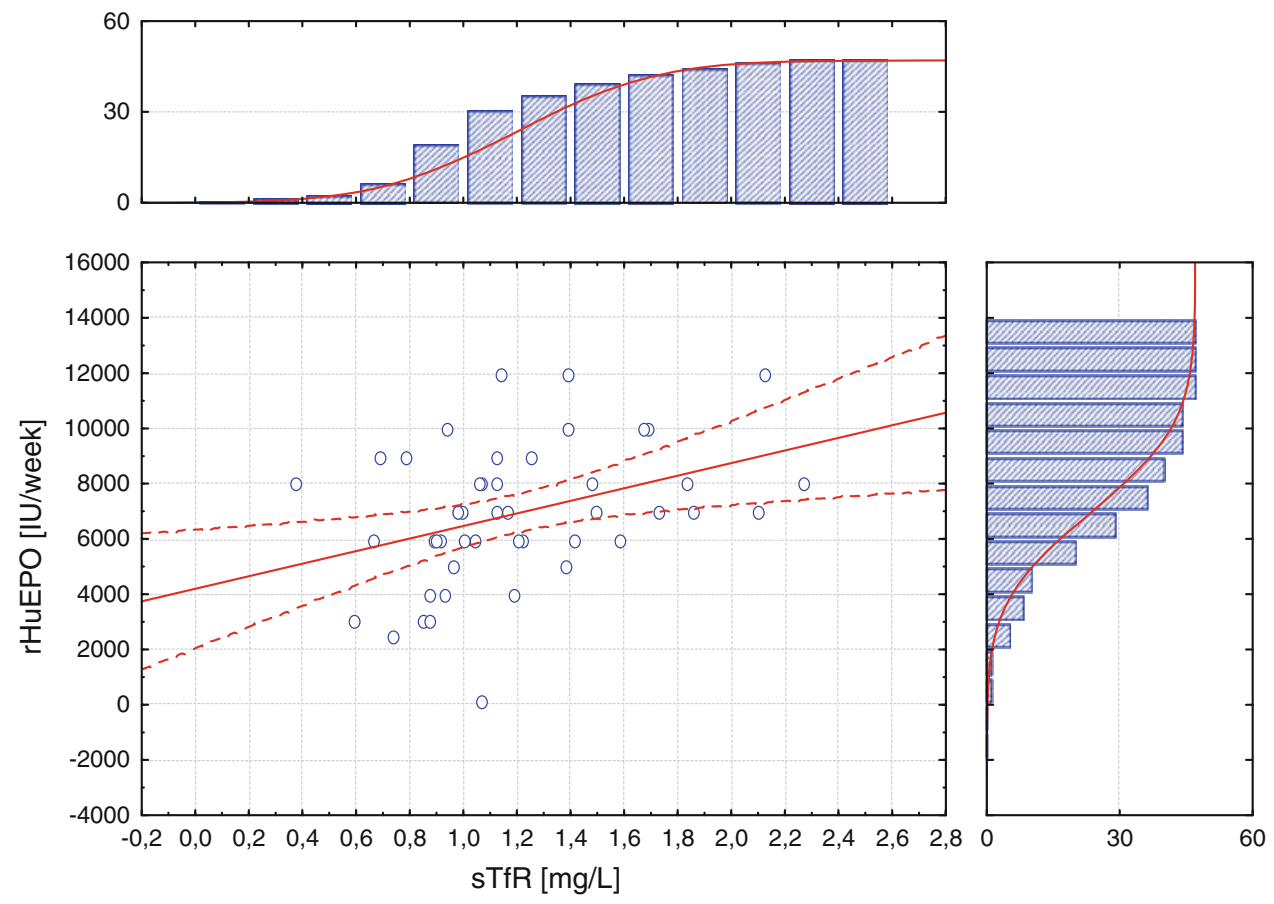
Fig. 5 The scatter diagram (with histograms, i.e., the upper and the right panel, which are a graphical way of presenting the empirical distribution characteristics) shows the association between sTfR and $\mathrm{Fe}$. It illustrates the strong negative correlation (Spearman rank correlation coefficient $r=-0.4284, p=0.004)$, which suggests a strong inverse relationship between these variables. The solid line indicates the linear regression, and the dotted line - the 95\% confidence interval. sTfR soluble transferrin receptor whose serum concentration is proportional to the total concentration of cellular TfR, $\mathrm{Fe}$ serum iron, $r$ Pearson's correlation coefficient, $p$ value of the significance level

Fig. 6 The scatter diagram (with histograms, i.e., the upper and the right panel, which are a graphical way of presenting the empirical distribution characteristics) shows the association between hepcidin and rHuEPO. It illustrates the strong negative correlation (Spearman rank correlation coefficient $r=-0.8368$, $p=0.000$ ), which suggests a strong inverse relationship between these variables. The solid line indicates the linear regression, and the dotted linethe $95 \%$ confidence interval. rHuEPO mean weekly dose of recombinant human erythropoietin, $r$ Pearson's correlation coefficient, $p$ value of the significance level
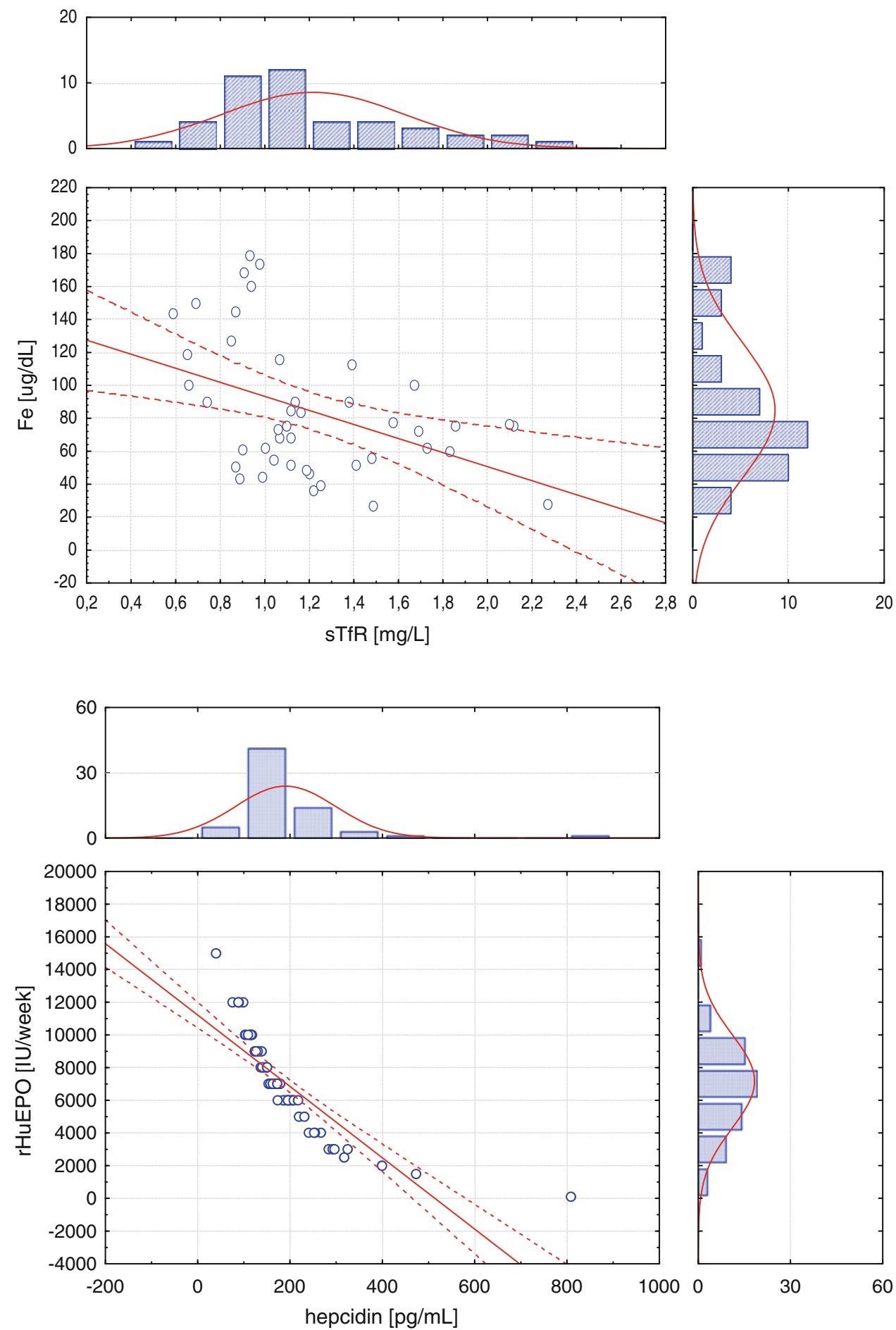

We subjected the obtained results for statistical analysis using Statistica 8.0 (Stat Soft Inc., Tulsa, USA). The significance level was assumed to be equal to 0.05 .

Analysis of the results of the laboratory tests

The lack of the statistically significant correlations (see Table 8) between sTfR and selected inflammatory variables confirms the results obtained on the basis of the analysis of the behaviour of our model, i.e., there is no connection between TfRs (transferrin receptors) and the inflammatory process.

Based on the lab studies, we assessed the impact of rHuEPO therapy on the concentration of sTfR. We have obtained results (see Fig. 4) confirming our previous observations made on the models' behavior, i.e., the 
existence of a positive correlation between STfR and dosage of rHuEPO.

Furthermore, as well as in our theoretical analysis, we found in lab analysis the relationship between low levels of serum iron and high levels of sTfR (Fig. 5). Other detected dependencies, i.e., between hepcidin and sTfR and also between serum EPO and hepcidin were in the lab test, not statistically significant, although similar trends like in behaviour of our model were preserved-an inverse relationship.

In addition, during the lab tests we confirmed our results based on the analysis of the model concerning the statistically significant existence of a relationship between serum hepcidin and the dose of rHuEPO (Fig. 6).

\section{Summary}

Anemia of chronic disorders is a very complex issue whose diagnosis is a not well-established procedure. To better understand this process and its influence on some other factors, a mathematical model of the body iron homeostasis has been built. The model has been formulated in the language of Petri net theory, which allows for its simulation and precise analysis, which, in turn, allows to answer some questions. On the basis of the net analyzed in this paper, we have found that there is no influence of inflammation on the concentration of the transferrin receptor. Moreover, the results of the model analysis suggest that erythropoietin and $\mathrm{rHuEPO}$ can influence the transferrin receptor levels. It was positively verified that $\mathrm{TfR}$ is a good marker of the erythropoietic activity. Also answers to questions if EPO influences the hepcidin concentration and how the concentration of hepcidin, HFE and TfR is changing during an inflammatory process have been found. The results obtained from the analysis of the model and the clinical research complement each other.

Acknowledgments We are very grateful to the anonymous referee who has helped us to improve the paper considerably. This research has been partially supported by the Polish Ministry of Science and Higher Education grants No. N N402 209833 and No. N N519 314635 .

Open Access This article is distributed under the terms of the Creative Commons Attribution Noncommercial License which permits any noncommercial use, distribution, and reproduction in any medium, provided the original author(s) and source are credited.

\section{References}

1. Hentze MW, Muckenthaler MU, Andrews NC (2004) Balancing acts: molecular control of mammalian iron metabolism. Cell $117: 285-297$
2. Amore A, Coppo R (2002) Immunological basis of inflammation in dialysis. Nephrol Dial Transpl 17:16-24

3. Macdougall IC, Cooper AC (2005) Hyporesponsiveness to erythropoietic therapy due to chronic inflammation. Eur J Clin Invest 35:32-35

4. Stenvinkel P, Bárány P (2002) Anemia, rHuEPO resistance, and cardiovascular disease in end-stage renal failure: links to inflammation and oxidative stress. Nephrol Dial Transpl 17:32-37

5. Tarng DC, Huang TP, Chen TW, Yang WC (1999) Erythropoietin hyporesponsiveness: from iron deficiency to iron overload. Kidney Int 69:107-118

6. Petri CA. (1962) Communication with automata (in German). Schriften des IIM Nr. 3, Institut für Instrumentelle Mathematik, Bonn

7. Reddy VN, Mavrovouniotis ML, Liebman MN (1993) Petri net representation in metabolic pathways. Proc Int Conf Intell Syst Mol Biol 1:328-336

8. Matsuno H, Li C, Miyano S (2006) Petri net based descriptions for systematic understanding of biological pathways. IEICE Trans Fundam Electron Commun Comput Sci E89-A:3166-3174

9. Voss K, Heiner M, Koch I (2003) Steady state analysis of metabolic pathways using Petri nets. In Silico Biol 3:367-387

10. Doi A, Fujita S, Matsuno H, Nagasaki M, Miyano S (2004) Constructing biological pathway models with hybrid functional Petri nets. In Silico Biol 4:271-291

11. Chaouiya $C$ (2007) Petri net modelling of biological networks. Brief Bioinform 8:210-219

12. Hardy S, Robillard PN (2004) Modeling and simulation of molecular biology systems using Petri nets: modeling goals of various approaches. J Bioinform Computat Biol 2:595-613

13. Peleg M, Rubin D, Altman RB (2005) Using Petri net tools to study properties and dynamics of biological systems. J Am Med Inform Assoc 12:181-199

14. Matsuno H, Tanaka Y, Aoshima H, Doi A, Matsui M, Miyano S (2003) Biopathways representation and simulation on hybrid functional Petri net. In Silico Biol 3:389-404

15. David R, Alla H (2005) Discrete continuous and hybrid Petri nets. Springer, Berlin

16. Murata T (1989) Petri nets: properties, analysis and applications. Proc IEEE 77:541-580

17. Sackmann A, Heiner M, Koch I (2006) Application of Petri net based analysis techniques to signal transduction pathways. BMC Bioinform 7:482

18. Sackmann A, Formanowicz D, Formanowicz P, Koch I, Błażewicz J (2007) An analysis of the Petri net based model of the human body iron homeostasis process. Comput Biol Chem $31: 1-10$

19. Formanowicz D, Sackmann A, Formanowicz P, Blazewicz J (2006) Petri net based model of the body iron homeostasis. J Biomed Inform 40:476-485

20. Błażewicz J, Formanowicz D, Formanowicz P, Sackmann A, Sajkowski M (2009) Modeling the process of human body iron homeostasis using a variant of timed Petri nets. Discrete Appl Math 157:2221-2231

21. Williams P, Griffiths E (1992) Bacterial transferrin receptorsstructure, function and contribution to virulence. Med Microbiol Immun 181:301-322

22. Ward CG, Bullen JJ, Rogers HJ (1996) Iron and infection: new developments and their implications. J Trauma 41:356-364

23. Deicher R, Horl WH (2004) Hepcidin: a molecular link between inflammation and anaemia. Nephrol Dial Transpl 19:521-524

24. Nemeth E, Tuttle M, Powelson J, Vaughn M, Donovan A, Ward D, Ganz T, Kaplan J (2004) Hepcidin regulates cellular iron efflux by binding to ferroportin and inducing its internalization. Science 306:2090-2093 
25. Frazer DM, Anderson GJ (2003) The orchestration of body iron intake: how and where do enterocytes receive their cues? Blood Cells Mol Dis 30:288-297

26. Starke PH (1990) Analysis of Petri net models (in German). Teubner, Stuttgart

27. Sackmann A, Formanowicz D, Formanowicz P, Błażewicz J (2009) New insights into the human body iron metabolism analysed by a Petri net based approach. Biosystems 96:104-113

28. Heiner M, Koch I, Will J (2004) Model validation of biological pathways using Petri nets-demonstrated for apoptosis. Biosystems 75:15-28

29. Grafahrend-Belau E, Schreiber F, Heiner M, Sackmann A, Junker BH, Grunwald S, Speer A, Winder K, Koch I (2008) Modularization of biochemical networks based on classification of Petri net t-invariants. BMC Bioinform 9:90

30. Formanowicz D, Pietrzak I (2006) The diagnostic value of soluble transferrin receptor in haemodialysed patients. Nowiny Lekarskie 75:421-425

31. Chiang WC, Tsai TJ, Chen YM, Lin SL, Hsieh BS (2002) Serum soluble transferrin receptor reflects erythropoiesis but not iron availability in erythropoietin-treated chronic hemodialysis patients. Clin Nephrol 58:363-369

32. Huebers HA, Beguin Y, Pootrakul P, Einspahr D, Finch CA (1990) Intact transferrin receptors in human plasma and their relation to erythropoiesis. Blood 75:102-107

33. Kohgo $Y$, Nishisato $T$, Kondo $H$, Tsushima N, Nitsu $Y$, Urushizaki I (1986) Circulating transferrin receptor in human serum. Brit J Haematol 64:277-281
34. Nakanishi T, Moriguchi R, Itahana R, Otaki Y, Inoue T, Takamitsu Y (2001) Acute effects of human recombinant erythropoietin administration on soluble transferrin receptor. Nephron 89:119-120

35. Ahluwalia N, Skikne BS, Savin V, Chonko A (1997) Markers of masked iron deficiency and effectiveness of EPO therapy in chronic renal failure. Am J Kidney Dis 30:532-541

36. Assandri R, Egger M, Gassmann M, Niggli E, Bauer C, Forstler I, Gorlach A (1999) Erythropoietin modulates intracellular calcium in a human neuroblastoma cell line. J Physiol 516:343-352

37. Chen $\mathrm{CH}$, Cheng $\mathrm{CH}$, Cheng TC, Shu KH, Wu MJ, Lian JD (1998) Serum transferrin receptor is a good index of erythropoietin treatment in ESRD. Acta Nephrol 12:7-11

38. Roque ME, Sandoval MJ, Aggio MC (2001) Serum erythropoietin and its relation with soluble transferrin receptor in patients with different types of anaemia in a locally defined reference population. Clin Lab Haematol 23:291-295

39. Formanowicz D (2005) The meaning of the transferrin glycosylation and the concentration of the soluble transferrin receptor as factors having an influence on anemia in patients with endstage renal disease treated by maintenance hemodialysis (in Polish). Doctoral dissertation, Poznań University of Medical Sciences, Poznań 